\title{
FUNCTIONS ON NONCOMPACT LIE GROUPS WITH POSITIVE FOURIER TRANSFORMS
}

\author{
TAKESHI KAWAZOE
}

(Communicated by J. Marshall Ash)

\begin{abstract}
Let $G$ be a homogeneous group with the graded Lie algebra or a noncompact semisimple Lie group with finite center. We define the Fourier transform $\hat{f}$ of $f$ as a family of operators $\hat{f}(\pi)=\int_{G} f(x) \pi(x) d x(\pi \in \widehat{G})$, and we say that $\hat{f}$ is positive if all $\hat{f}(\pi)$ are positive. Then, we construct an integrable function $f$ on $G$ with positive $\hat{f}$ and the restriction of $f$ to any ball centered at the origin of $G$ is square-integrable, however, $f$ is not square-integrable on $G$.
\end{abstract}

\section{INTRODUCTION}

When $G$ is a compact abelian group, integrable functions $f$ on $G$ with the nonnegative Fourier coefficients and being $p$ th $(1<p \leq 2)$ power integrable near the identity of $G$ have the Fourier coefficients in $l^{q}(q=p /(p-1))$. This result was first obtained by N. Wiener in the case of $G=\mathbf{T}$ and $p=2$ (cf. [3]) and then by Rains [9] and Ash, Rains, and Vági [1] for arbitrary compact abelian groups. When $G$ is a compact semisimple Lie group, an analogous result was obtained by the author and Miyazaki [6]. Furthermore, Nassiet [8] and Blank [2] treated the same problem in the case that $G$ is a compact separable group.

When $G$ is not compact, for example, when $G=\mathbf{R}$, a counterexample was obtained by the author, Onoe, and Tachizawa [7]: there exists an integrable function $f$ on $\mathbf{R}$ with nonnegative Fourier transform and the restriction of $f$ to a neighborhood of 0 is square-integrable, however, $f$ is not square-integrable on $\mathbf{R}$. In this paper we shall also give a counterexample when $G$ is a homogeneous group with the graded Lie algebra (see [4, Chapter 1]) and also when $G$ is a noncompact semisimple Lie group with finite center. In the case of a homogeneous group with the graded Lie algebra we can find a one-parameter subgroup $\mathscr{A}$ of $G$ for which $a x a^{-1}=x$ for all $a \in \mathscr{A}$ and $x \in G$. Then, regarding $\mathscr{A}$ as $\mathbf{R}$, we can apply the same idea used in [7] to construct the counterexample on $G$. As compared with [7], our proof is simple and group-theoretical. Especially, the condition (3) in [7] can be replaced by a weaker condition. In the case of a noncompact semisimple Lie group we can take a one-parameter subgroup $\mathscr{A}$ of $G$ as a subgroup of the maximal abelian subgroup $A$ of $G$. Although $\mathscr{A}$

Received by the editors July 7, 1993.

1991 Mathematics Subject Classification. Primary 42A38; Secondary 42C15. 
does not belong to the center of $G$, the same idea is still applicable to obtain a counterexample.

\section{HOMOGENEOUS GROUPS}

2.1. Notation. Let $G$ be a homogeneous group whose Lie algebra $\mathfrak{g}$ is graded and $|\cdot|: G \rightarrow \mathbf{R}_{+}$a homogeneous norm of $G$ (see [4, Chapter 1]). $\mathfrak{g}$ is endowed with a vector space decomposition $\mathfrak{g}=\sum_{k=1}^{\infty} V_{k}$ such that $\left[V_{i}, V_{j}\right] \subset V_{i+j}$, where all but finitely many $V_{k}$ 's are $\{0\}$. Then if we take $k_{0}=\max \left\{k ; V_{k} \neq\right.$ $\{0\}\}$ and $X \neq 0 \in V_{k_{0}}, \mathscr{A}=\exp (\mathbf{R} X)$ satisfies

$$
a x a^{-1}=x \text { for all } a \in \mathscr{A} \text { and } x \in G \text {. }
$$

Some examples may be in order: (i) Noncompact abelian groups $\mathbf{R}^{n}$; (ii) Heisenberg group $H_{n}$. The underlying manifold is $\mathbf{C}^{n} \times \mathbf{R}$ and the multiplication law is given as

$$
\begin{aligned}
& \left(z_{1}, \ldots, z_{n}, t\right)\left(z_{1}^{\prime}, \ldots, z_{n}^{\prime}, t^{\prime}\right) \\
& \quad=\left(z_{1}+z_{1}^{\prime}, \ldots, z_{n}+z_{n}^{\prime}, t+t^{\prime}+2 \mathfrak{I}\left(\sum_{j=1}^{n} z_{j} \bar{z}_{j}^{\prime}\right)\right) .
\end{aligned}
$$

Then $\mathscr{A}=(0, \ldots, 0, \mathbf{R})$ satisfies $(*)$; and (iii) The group of all upper triangle matrices $\left(a_{i j}\right)_{1 \leq i, j \leq n}$ with $a_{j j}=1 \quad(1 \leq j \leq n)$. Then $\mathscr{A}=\exp \left(\mathbf{R} E_{1 n}\right)$ satisfies $(*)$, where $E_{1 n}$ is the matrix with 0 entries but 1 in the $(1, n)$ entry. Let $d x$ be a $G$-invariant measure on $G$. We denote the volume of a measurable set $S$ of $G$ by $|S|$ and the $L^{p}$-norm $(1 \leq p<\infty)$ of a function $f$ on $G$ by $\|f\|_{p}=\left(\int_{G}|f(x)|^{p} d x\right)^{1 / p}$. For any integrable functions $f$ on $G$ we denote the Fourier transform $\hat{f}$ of $f$ as a family of operators $\hat{f}(\pi)=\int_{G} f(x) \pi(x) d x$ $(\pi \in \widehat{G})$. We say that $\hat{f}$ is positive if all $\hat{f}(\pi) \quad(\pi \in \widehat{G})$ are positive operators (see [11, p. 317]), which we denote by $\hat{f}(\pi) \geq 0$. Let $B(r)=\{x \in G ;|x| \leq r\}$ $\left(r \in \mathbf{R}_{+}\right)$. Then there exists a positive constant $D$ such that

$$
|B(r)| \sim r^{D} \quad\left(r \in \mathbf{R}_{+}\right)
$$

(see [4, p. 10]). Let $\left\{a_{n}\right\}_{n \in \mathbf{N}}$ be a sequence in $\mathscr{A}$ such that $\left|a_{n}\right|=n$, and let $\left\{b_{n}\right\}_{n \in \mathbf{N}}$ and $\left\{r_{n}\right\}_{n \in \mathbf{N}}$ be sequences in $\mathbf{R}_{+}$satisfying

(1) $r_{1}<1 / 2, r_{n}$ is decreasing, and there exists $L \in \mathbf{R}_{+}$such that $r_{n} \geq 2 r_{m}$ $(m \geq n)$ if and only if $m \geq L n$,

(2) $\sum_{n=1}^{\infty} b_{n}\left|B\left(r_{n}\right)\right|<\infty$,

(3) for each $M \in \mathbf{R}_{+}, \sum_{n=1}^{\infty} \sum_{m \in \mathbf{N},|n-m| \leq M} b_{n} b_{m}\left|B\left(r_{n}\right)\right|^{1 / 2}\left|B\left(r_{m}\right)\right|<\infty$,

(4) for each $N \in \mathbf{R}_{+}$,

$$
\sum_{n=1}^{\infty} \sum_{n^{\prime}=n}^{\infty} \sum_{l \geq N n^{\prime}}^{\infty} b_{n} b_{n^{\prime}} b_{n+l} b_{n^{\prime}+l}\left|B\left(r_{n^{\prime}}\right)\left\|B\left(r_{n+l}\right)\right\| B\left(r_{n^{\prime}+l}\right)\right|=\infty .
$$

Example. Let $(\alpha, \beta)$ be a pair of positive numbers satisfying (i) $\alpha-\beta+1<0$, (ii) $4 \alpha-3 \beta+2<0$, and (iii) $4 \alpha-3 \beta+3 \geq 0$. For instance, $\alpha=3$ and $\beta=5$. Here we let $b_{n}=n^{\alpha}$ and $\left|B\left(r_{n}\right)\right|=n^{-\beta}$. Then (1) is obvious and (2) 
follows from (i). For (3) it is enough to estimate the sum of $n$ over $2 M$ and then

$$
\sum_{n=2 M}^{\infty} \sum_{\substack{m \in \mathbf{N} \\|n-m| \leq M}} n^{\alpha} m^{\alpha} n^{-\beta / 2} m^{-\beta} \leq c \sum_{n=2 M}^{\infty} n^{2 \alpha-3 \beta / 2}<\infty
$$

by (ii). (4) follows from (iii) as

$$
\begin{aligned}
\sum_{n=1}^{\infty} \sum_{n^{\prime}=n}^{\infty} \sum_{l \geq N n^{\prime}}^{\infty} n^{\alpha} n^{\alpha}(n+l)^{\alpha}\left(n^{\prime}+l\right)^{\alpha} n^{\prime-\beta}(n+l)^{-\beta}\left(n^{\prime}+l\right)^{-\beta} \\
\quad \geq \sum_{n=1}^{\infty} \sum_{n^{\prime}=n}^{\infty} n^{\alpha} n^{\alpha-\beta} \int_{N n^{\prime}}^{\infty}(n+x)^{\alpha-\beta}\left(n^{\prime}+x\right)^{\alpha-\beta} d x \\
\quad \geq \sum_{n=1}^{\infty} \sum_{n^{\prime}=n}^{\infty} n^{\alpha} n^{\prime 3 \alpha-3 \beta+1} \int_{N}^{\infty}(x+1)^{2 \alpha-2 \beta} d x \\
\quad \geq c \sum_{n=1}^{\infty} n^{\alpha} \int_{n}^{\infty} x^{3 \alpha-3 \beta+1} d x \\
\quad \geq c \sum_{n=1}^{\infty} n^{4 \alpha-3 \beta+2}=\infty
\end{aligned}
$$

2.2. Counterexample. For each measurable set $S$ of $G$ we denote by $\chi_{S}$ the characteristic function of $S$. Now we define a function $g_{n}(n \in \mathbf{N})$ on $G$ as $g_{n}(x)=b_{n} \mathbf{x}_{n}(x)=b_{n} \chi_{B\left(r_{n}\right)}\left(a_{n}^{-1} x\right) \quad(x \in G)$ and put $g=\sum_{n=1}^{\infty} g_{n}$. Then, $\|g\|_{1}=\sum_{n=1}^{\infty}\left\|g_{n}\right\|_{1}=\sum_{n=1}^{\infty} b_{n}\left|B\left(r_{n}\right)\right|<\infty$ by (2). Here we let $f=g^{\sim} * g$, where $g^{\sim}(x)=g\left(x^{-1}\right)$. Then

(5) $\|f\|_{1} \leq\left\|g^{\sim}\right\|_{1}\|g\|_{1}=\|g\|_{1}^{2}<\infty \quad$ and $\hat{f}(\pi)=\hat{g}\left(\pi^{*}\right) \hat{g}(\pi) \geq 0 \quad(\pi \in \hat{G})$.

Since $\operatorname{supp}\left(\mathbf{x}_{n}^{\sim} * \mathbf{x}_{m}\right)=B\left(r_{n}\right) a_{m-n} B\left(r_{m}\right) \subset B\left(r_{1}\right) a_{m-n} B\left(r_{1}\right)$ (see (1)), it follows that $g_{n}^{\sim} * g_{m}(x)=0$ if $x \in B(R) \quad\left(R \in \mathbf{R}_{+}\right)$and $|m-n|>2 r_{1}+R$. Therefore, we can deduce that for each $R \in \mathbf{R}_{+}$

$$
\begin{aligned}
\left(\int_{B(R)}|f(x)|^{2} d x\right)^{1 / 2} & \leq \sum_{n=1}^{\infty} \sum_{\substack{m \in \mathbf{N} \\
|n-m| \leq 2 r_{1}+R}}\left\|g_{n}^{\sim} * g_{m}\right\|_{2} \\
& \leq \sum_{n=1}^{\infty} \sum_{\substack{m \in \mathbf{N} \\
|n-m| \leq 2 r_{1}+R}}\left\|g_{n}\right\|\left\|_{2}\right\| g_{m} \|_{1} \\
& =\sum_{n=1}^{\infty} \sum_{\substack{m \in \mathbf{N} \\
|n-m| \leq 2 r_{1}+R}} b_{n} b_{m}\left|B\left(r_{n}\right)\right|^{1 / 2}\left|B\left(r_{m}\right)\right|<\infty
\end{aligned}
$$

by (3). Finally we show that $\|f\|_{2}=\infty$. Since

$$
\mathbf{x}_{n}^{\sim} * \mathbf{x}_{m}(x)=\left|a_{n} B\left(r_{n}\right) x \cap a_{m} B\left(r_{m}\right)\right|=\left|a_{m-n}^{-1} B\left(r_{n}\right) x \cap B\left(r_{m}\right)\right| \quad(x \in G),
$$

it follows from $(*)$ that if $m \geq n$ and $x \in a_{m-n} B\left(r_{n}-r_{m}\right)$, then $a_{m-n}^{-1} B\left(r_{n}\right) x \supset$ $B\left(r_{m}\right)$ and thus, $\mathbf{x}_{n}^{\sim} * \mathbf{x}_{m}(x)=\left|B\left(r_{m}\right)\right|$. On the other hand, (1) implies that there exists $N=L-1 \in \mathbf{R}_{+}$such that $r_{n}-r_{m} \geq r_{n} / 2$ if and only if $m \geq(N+1) n$. 
Therefore, we can deduce that if $m \geq(N+1) n$, then $\mathbf{x}_{n}^{\sim} * \mathbf{x}_{m}(x)=\left|B\left(r_{m}\right)\right|$ for $x \in B\left(r_{n} / 2\right)$. Since $|B(r)| \sim|B(2 r)|$ by $(* *)$, it follows from (4) that

$$
\begin{aligned}
\|f\|_{2}^{2} & =g^{\sim} * g *\left(g^{\sim} * g\right)^{\sim}(1) \\
& \geq \sum_{n=1}^{\infty} \sum_{n^{\prime}=n}^{\infty} \sum_{l \geq N n^{\prime}}^{\infty} b_{n} b_{n^{\prime}} b_{n+l} b_{n^{\prime}+l} \mathbf{x}_{n}^{\sim} * \mathbf{x}_{n+l} * \mathbf{x}_{n^{\prime}}^{\sim} * \mathbf{x}_{n^{\prime}+l}(1) \\
& \geq \sum_{n=1}^{\infty} \sum_{n^{\prime}=n}^{\infty} \sum_{l \geq N n^{\prime}}^{\infty} b_{n} b_{n^{\prime}} b_{n+l} b_{n^{\prime}+l}\left|B\left(r_{n^{\prime}}\right)\left\|B\left(r_{n+l}\right)\right\| B\left(r_{n^{\prime}+l}\right)\right|=\infty,
\end{aligned}
$$

where the symbol " $\sim$ " means that the ratio of the right- and left-hand sides is bounded below and above by positive constants. Then (5)-(7) implies the following

Theorem 1. Let $G$ be a homogeneous group with graded Lie algebra. Then there exists an integrable function $f$ on $G$ with positive $\hat{f}$ and the restriction of $f$ to any ball centered at the origin of $G$ is square-integrable, however, $f$ is not square-integrable on $G$.

\section{SEMISIMPLE LIE GROUPS}

3.1. Notation. Let $G$ be a noncompact semisimple Lie group with finite center and $G=K C L\left(A_{+}\right) K$ a Cartan decomposition of $G$. Let $\sigma: G \rightarrow \mathbf{R}_{+}$denote the $K$-bi-invariant function on $G$ defined by $\sigma(x)=d(\overline{1}, \bar{x}) \quad(x \in G)$, where $d$ is the Riemannian distance on the symmetric space $X=G / K, x \rightarrow \bar{x}$ is the natural map of $G$ to $X$, and 1 is the origin of $G$ (cf. [10]). Let $\mathscr{A}=\left\{a_{t} \in\right.$ $A ; t \in \mathbf{R}\}$ be a one-parameter subgroup of $G$ for which $\left\{a_{n}\right\}_{n \in \mathbf{N}}$ is a sequence in $A_{+}$such that $\sigma\left(a_{n}\right)=n$. Let $d x$ be a $G$-invariant measure on $G$. As in the case of homogeneous groups, we define the volume $|S|$ of a measurable set $S$ of $G$, the $L^{p}$-norm $\|f\|_{p}$, and the Fourier transform $\hat{f}$ of a function $f$ on $G$. Let $B(r)=\{x \in G ; \sigma(x) \leq r\}$. Then there exists a positive constant $D$ such that

$(* * *)$

$$
|B(r)| \sim r^{D} \quad(r<1)
$$

(cf. [5, Chapter X]). We fix two sequences $\left\{b_{n}\right\}_{n \in \mathbf{N}}$ and $\left\{r_{n}\right\}_{n \in \mathbf{N}}$ in $\mathbf{R}_{+}$satisfying the exactly same conditions (1)-(4).

3.2. Counterexample. We define a right $K$-invariant function $g_{n}(n \in \mathbf{N})$ as $g_{n}(x)=b_{n} \mathbf{x}_{n}(x)=b_{n} \chi_{B\left(r_{n}\right)}\left(a_{n}^{-1} x\right) \quad(x \in G)$. We put $g=\sum_{n=1}^{\infty} g_{n}$ and define a $K$-bi-invariant function $f$ on $G$ as $f=g^{\sim} * g$. By the same arguments which yield (5) and (6), we see that $f \in L^{1}(G), \hat{f} \geq 0$, and $\left.f\right|_{B(R)} \in L^{2}(G)$ for each $R \in \mathbf{R}_{+}$. Now we show that $\|f\|_{2}=\infty$. Although (*) does not hold for $\mathscr{A}$, it follows that

$$
(* * * *) \quad a B(r) a^{-1} K \supset B(r) \quad \text { for all } a \in A \text { and } r \in \mathbf{R}_{+} \text {. }
$$

Therefore, if $m \geq n$ and $x=a_{m-n} z \in a_{m-n} B\left(r_{n}-r_{m}\right)$, we can deduce that $a_{m-n}^{-1} B\left(r_{n}\right) \bar{x} \supset B\left(r_{n}\right) \bar{z} \supset B\left(r_{m}\right)$; and thus, applying the same argument used in the case of homogeneous groups, we can obtain that $\|f\|_{2}=\infty$. 
Theorem 2. Let $G$ be a noncompact semisimple Lie group with finite center. Then there exists an integrable $K$-bi-invariant function $f$ on $G$ with positive $\hat{f}$ and the restriction of $f$ to any ball centered at the origin of $G$ is squareintegrable, however, $f$ is not square-integrable on $G$.

In the proofs of Theorems 1 and 2 the structure of Lie groups is not essential. Actually, let $G$ be a noncompact separable group and suppose that $G$ has a oneparameter subgroup $\mathscr{A}=\left\{a_{t} ; t \in \mathbf{R}\right\}$ of $G$ and the family of neighborhoods of the identity of $G$ parametrized as $B(r) \quad(0<r \leq 1)$ satisfying (i) $B(r) \subset B\left(r^{\prime}\right)$ if $r<r^{\prime}$, (ii) $|B(r)| \sim r^{D}$ for $D>0$, (iii) $a_{n} B(1) \quad(n \in \mathbf{N})$ are disjoint, and (iv) $a B(r) a^{-1} \supset B(r)$ for all $a \in \mathscr{A}$ and $0<r \leq 1$. Then, by the same argument used in the proof of Theorem 2, we can construct the counterexample for $G$.

\section{REFERENCES}

1. J. M. Ash, M. Rains, and S. Vági, Fourier series with nonnegative coefficients, Proc. Amer. Math. Soc. 101 (1987), 392-393.

2. B. E. Blank, Fourier decompositions and positive coefficients in compact Gelfand pairs, Proc. Amer. Math. Soc. 119 (1993), 427-430.

3. R. P. Boas, Entire functions, Academic Press, New York, 1964.

4. G. B. Folland and E. M. Stein, Hardy spaces on homogeneous groups, Math. Notes, no. 28, Princeton Univ. Press, Princeton, NJ, 1982.

5. S. Helgason, Differential geometry and symmetric spaces, Academic Press, New York, 1962.

6. T. Kawazoe and H. Miyazaki, Fourier series with nonnegative coefficients on compact semisimple Lie groups, Tokyo J. Math. 12 (1989), 241-246.

7. T. Kawazoe, Y. Onoe, and K. Tachizawa, Functions on $\mathbf{R}$ with nonnegative Fourier transforms, Tôhoku Math. J. 46 (1994), 311-320.

8. F. Nassiet, Séries de Fourier à coefficients positifs, C. R. Acad. Sci. Paris Sèr. I 313 (1991), $1-4$.

9. M. Rains, On functions with nonnegative Fourier transforms, Indian J. Math. 27 (1985), 41-48.

10. V. S. Varadarajan, Harmonic analysis on reductive groups, Lecture Notes in Math., vol. 576, Springer-Verlag, New York, 1977.

11. K. Yosida, Functional analysis, second ed., Springer-Verlag, New York, 1968.

Departement de Mathematiques, Universite de Nancy I, 54506 Vandoeuvre les Nancy, FRANCE

Current address: Department of Mathematics, Faculty of Science and Technology, Keio University, 3-14-1, Hiyoshi, Kohokuku, Yokohama 223, Japan

E-mail address: kawazoe@math.keio.ac.jp 